J. Austral. Math. Soc. 21 (1976) (Series A), 234-240.

\title{
CHAINS IN GENERALIZED BOOLEAN LATTICES
}

\author{
RICHARD D. BYRD and ROBERTO A. MENA
}

(Received 4 June 1974; revised 4 March 1975)

Communicated by T. E. Hall

\section{Introduction}

A chain $C$ in a distributive lattice $L$ is called strongly maximal in $L$ if and only if for any homomorphism $\phi$ of $L$ onto a distributive lattice $K$, the chain $(C \phi)^{0}$ is maximal in $K$, where $(C \phi)^{0}=C \phi$ if $0 \notin K$, and $(C \phi)^{0}=C \phi \cup\{0\}$, otherwise. Gratzer (1971, Theorem 28) states that if $B$ is a generalized Boolean lattice $R$-generated by $L$ and $C$ is a chain in $L$, then $C R$-generates $B$ if and only if $C$ is strongly maximal in $L$. In this note (Theorem 4.6), we prove the following assertion, which is not far removed from Gratzer's statement:

let $B$ be a generalized Boolean lattice $R$-generated by $L$ and $C$ be a chain in $L$. If $0 \in L$, then $C$ generates $B$ if and only if $C$ is strongly maximal in $L$. If $0 \notin L$, then $C$ generates $B$ if and only if $C$ is strongly maximal in $L$ and $[C)_{L}=L$.

In Section 5 (Example 5.1) a counterexample to Gratzer's statement is provided.

In Section 3 (Theorem 3.6) we prove that there is a one-to-one mapping of the prime ideals of $L$ into the prime ideals of $B$, where $B$ is a generalized Boolean lattice generated by $L$, and (Corollary 3.7) that this mapping is onto if and only if $0 \in L$. In Section 4 (Proposition 4.3 and Corollary 4.7) we give sufficient conditions on a chain $C$ of $L$ so that $C \cup\{0\}$ is maximal in $B$.

The authors would like to thank the referee for his useful suggestions.

\section{Preliminaries}

For the standard results and definitions concerning lattices, the reader is referred to Gratzer (1971), particularly to Sections 9 and 10 of Chapter 2. Throughout this note, $B$ will denote a generalized Boolean lattice with smallest element 0 and $L$ will denote a sublattice of $B$ that generates $B$, that is, the smallest subring of $B$ that contains $L$ is $B$. If $E=$ $\left\{a_{1}+\cdots+a_{2 n} \mid a_{1}, \cdots, a_{2 n} \in L\right\}$, then $E$ is an ideal of $B$, called the ideal of $B$ evenly generated by $L$, and $E=\left\{a_{1}+\cdots+a_{2 n} \mid a_{1}, \cdots, a_{2 n} \in L\right.$ and $a_{1} \leqq \cdots \leqq$ 
$\left.a_{2_{n}}\right\}$ Byrd, Mena and Troy (1975, Lemma 2.4). Moreover, it is shown by these authors in Theorem 2.4 that $0 \notin L$ if and only if $L \cap E$ is the empty set, and in this case, $E$ is a maximal ideal of $B$ and the only maximal ideal of $B$ whose intersection with $L$ is void, see Byrd, Mena and Troy (1975, Corollary 2.5).

We say that $L R$-generates $B$ if $L$ generates $B$ and if $L$ has a smallest element, then it is the zero of $B$. Thus, if $L$ does not have a smallest element, the definitions of generates and $R$-generates coincide. A chain in $L$ is a sublattice of $L$ which is linearly ordered. The set of natural numbers will be denoted by $N$, the collection of prime ideals of $L$ will be denoted by $\mathscr{P}(L)$, the empty set will be denoted by $\square$, the set of elements in the set $X$ but not in the set $Y$ will be denoted by $X \backslash Y$, and the power set of $X$ will be denoted by $p(X)$. Finally, $C_{2}$ will denote the two element lattice $\{0,1\}$.

\section{Prime ideals}

It is well known that the collection of prime ideals of $B$ is identical with the collection of maximal ideals of $B$, and hence, trivially ordered. For $P \in$ $\mathscr{P}(B) \backslash\{E\}$, the mapping $P \rightarrow P \cap L$ is easily seen to be a one-to-one mapping of $\mathscr{P}(B) \backslash\{E\}$ into $\mathscr{P}(L)$. (As noted above, if $0 \notin L$, then $L \cap E=\square$ and if $0 \in L, E=B$.) A way of proving that this mapping is onto, is to form the collection $\{Q \mid Q$ is an ideal of $B$ and $Q \cap L=J\}$, where $J \in \mathscr{P}(L)$, use Zorn's lemma to pick a maximal element in this collection, and then prove that this element is prime in $B$. In this section we explicitly give the inverse of this mapping without the use of Zorn's lemma.

In Propositions 3.1 through $3.5, J$ will denote a prime ideal of $L$, $E_{L \backslash J}=\left\{x \mid x \in B\right.$ and $x=a_{1}+\cdots+a_{2 n}$ for some $\left.a_{1}, \cdots, a_{2 n} \in L \backslash J\right\}$, and $P=(J]_{B}+E_{L \backslash J}=\left\{u+v \mid u \in(J]_{B}\right.$ and $\left.v \in E_{L \backslash J}\right\}$, where $(J]_{B}$ denotes the ideal of $B$ generated by $J$. According to Byrd, Mena and Troy (1975, Lemma 2.1), $E_{L \backslash J}=\left\{a_{1}+\cdots+a_{2 n} \mid a_{1}, \cdots, a_{2 n} \in L \backslash J\right.$ and $\left.a_{1} \leqq \cdots \leqq a_{2 n}\right\}$ and, since $L \backslash J$ is a sublattice of $L, E_{L \backslash J}$ is a subring of $B$.

Proposition 3.1. $P$ is an ideal of $B$.

Proof. Obviously, $P$ is a subgroup of $B$. Thus, to show that $P$ is an ideal of $B$, it suffices to show that if $a \in L$ and $v \in E_{L \backslash J}$, then $a v \in P$. If $a \in J$, then $a v \in(J]_{B} \subseteq P$. If $a \in L \backslash J$, then $a v \in E_{L \backslash J} \subseteq P$. Hence, $P$ is an ideal of $B$.

Proposition 3.2. $P \cap(L \backslash J)=\square$ and hence, $P \cap L=J$.

Proof. Suppose (by way of contradiction) that $a \in P \cap(L \backslash J)$. Then $a=u+a_{1}+\cdots+a_{2 n}$, where $u \in(J]_{B}$ and $a_{1}, \cdots, a_{2 n} \in L \backslash J$, with $a_{1} \leqq \cdots \leqq$ $a_{2 n}$. Thus, $a \cdot a_{1}=\left(u+a_{1}+\cdots+a_{2 n}\right) a_{1}=u \cdot a_{1}+2 n a_{1}=u \cdot a_{1} \leqq u$. But this is a contradiction, since $a \cdot a_{1} \notin J$. 
Proposition 3.3. $P=\left\{u \vee v \mid u \in(J]_{B}\right.$ and $\left.v \in E_{L \backslash J}\right\}$.

Proof Let $Q=\left\{u \vee v \mid u \in(J]_{B}\right.$ and $\left.v \in E_{L \backslash J}\right\}$. Then clearly $(J]_{B} \cup$ $E_{L \backslash J} \subseteq Q \subseteq P$ and $Q$ is a join semilattice of $B$. Let $x \in B$ with $x \leqq z$ for some $z \in Q$. Then $x \in P$ and $x=a_{1}+\cdots+a_{m}$, where $a_{1}, \cdots, a_{m} \in L$ and $a_{1} \leqq \cdots \leqq$ $a_{m}$. If $a_{1} \notin J$, then $a_{2}, \cdots, a_{m} \notin J$ and we assert that $m$ is even; for otherwise, $m$ is odd and since $x \in P, m>1$. But then, $a_{2}+\cdots+a_{m} \in E_{L \backslash J}$ and hence, $a_{1}=$ $x+a_{2}+\cdots+a_{m} \in P$, a contradiction. Thus, $m$ is even and so $x \in E_{\mathrm{L} \backslash J} \subseteq Q$. If $a_{m} \in J$, then $x \in(J]_{B} \subseteq Q$. Hence, we may suppose that for some $1 \leqq k<m$, $a_{1}, \cdots, a_{k} \in J \quad$ and $a_{k+1}, \cdots, a_{m} \in L \backslash J$. Consequently, $x_{1}=$ $a_{1}+\cdots+a_{k} \in(J]_{B} \subseteq Q \subseteq P$ and hence, $x_{2}=a_{k+1}+\cdots+a_{m}=x+x_{1} \in P$. It follows that $m-k$ is even and so $x_{2} \in E_{L \backslash J} \subseteq Q$. Therefore, $x_{1} x_{2}=$ $x_{1}\left(a_{k+1}+\cdots+a_{m}\right)=(m-k) x_{1}=0$. Hence, $x=x_{1}+x_{2}=x_{1}+x_{2}+x_{1} x_{2}=$ $x_{1} \vee x_{2} \in Q$. Thus, $Q$ is an ideal of $B$ that contains $(J]_{B} \cup E_{L \backslash}$, and so $Q=P$.

Proposition 3.4. If $L$ is linearly ordered, then $P$ is the direct sum of $(J]_{B}$ and $E_{L} \backslash$.

Proof. If $x \in(J]_{B} \cap E_{L \backslash J}$, then $x \leqq j$ for some $j \in J$ and $x=a_{1}+\cdots+a_{2 n}$ for some $a_{1}, \cdots a_{2 n} \in L \backslash J$. Since $L$ is linearly ordered, $j \leqq a_{i}$ for each $i$ and so $x=x j=\left(a_{1}+\cdots+a_{2 n}\right) j=2 n j=0$.

In Section 5 (Example 5.1) we show that, in general, $P$ is not the direct sum of $(J]_{B}$ and $E_{L \backslash J}$.

Proposition 3.5. $P \in \mathscr{P}(B)$.

Proof. If $x \in B \backslash P$, then $x=b_{1}+\cdots+b_{m}$, where $b_{1}, \cdots, b_{m} \in L$ and $b_{1} \leqq \cdots \leqq b_{m}$. Since $x \notin P, b_{m} \notin J$. If $b_{1} \notin J$, then $m$ is odd and $P+x=P+b_{m}$. Suppose that for some $1 \leqq k<m, b_{1}, \cdots, b_{k} \in J$ and $b_{k+1}, \cdots, b_{m} \in L \backslash J$. Then $m-k$ must be odd as $x \notin P$ and again $P+x=P+b_{m}$. Now if $a, b \in L \backslash J$, then $a+b \in P$ and it follows that the index of $P$ in $B$ is two. Hence, $P$ is a maximal ideal of $B$ and consequently, $P$ is prime.

Combining the above we now prove

THEOREM 3.6. The mapping $\nu$ of $\overline{\mathscr{P}}(\bar{L})$ into $\mathscr{P}(B)$ given by $J \nu=(J]_{B}+E_{L \backslash J}$ is a one-to-one mapping of $\mathscr{P}(L)$ into $\mathscr{P}(B)$. If $P \in \mathscr{P}(B) \backslash\{E\}$, then $P$ belongs to the range of $\nu$ and $P \nu^{-1}=P \cap L$.

Proof. By Proposition 3.5, $\nu$ is a mapping of $\mathscr{P}(L)$ into $\mathscr{P}(B)$. By Proposition 3.2, $\nu$ is one-to-one.

If $P \in \mathscr{P}(B) \backslash\{E\}$, then $P \cap L \in \mathscr{P}(L)$. Now $(P \cap L]_{B} \subseteq P$. If $a_{1}, a_{2} \in L \backslash P$ with $a_{1} \leqq a_{2}$, then $a_{1}\left(a_{1}+a_{2}\right)=0$. Since $P$ is prime, $a_{1}+a_{2} \in P$. Therefore, $E_{L \backslash(P \cap L)} \subseteq P$. Thus, $(P \cap L]_{B}+E_{L \backslash(P \cap L)} \subseteq P$ and by Proposition 3.5, we must have equality. Hence, $P$ belongs to the range of $\nu$ and $P \nu^{-1}=P \cap L$.

Corollary 3.7. $\nu$ is onto if and only if $0 \in L$. 
We close this section with the following proposition (see the Lemma in Makinson (1969) or the proof in Gratzer (1971, Theorem 28)).

Proposition 3.8. Let $A$ be a proper subring of $B$ and $x \in B \backslash A$. If $x<z$ for some $z \in A$, then there exists $P, Q \in \mathscr{P}(B)$ such that $x \in Q \backslash P, z \notin P \cup Q$, and $P \cap A=Q \cap A$. If, in addition, $x>$ a for some $a \in L \cap A$, then $P \cap Q \cap L \neq \square$.

\section{Chains}

If $C \subseteq L$, then let

$$
C^{0}= \begin{cases}C \cup\{a\} & \text { if } a \text { is the smallest element of } L, \\ C & \text { if } L \text { has no smallest element. }\end{cases}
$$

A chain $C$ of $L$ is said to be strongly maximal in $L$ if and only if for any homomorphism $\phi$ of $L$ onto a distributive lattice $K$, the chain $(C \phi)^{0}$ is maximal in $K$, see Gratzer (1971, page 114).

Proposition 4.1. If $L$ does not $R$-generate $B$, then $L$ contains an atom of $B$.

Proof. Since $L$ generates $B$ but does not $R$-generate $B, L$ must contain a smallest element $b>0$. Let $x \in B$ with $0 \leqq x \leqq b$. Then $x=a_{1}+\cdots+a_{m}$, where $a_{1}, \cdots, a_{m} \in L$ and $0<a_{1} \leqq \cdots \leqq a_{m}$. Then $x=x b=\left(a_{1}+\cdots+a_{m}\right) b=$ $m b$, as $b \leqq a_{1}$. If $m$ is even, then $m b=0$. If $m$ is odd, then $m b=b$. Whence, $b$ is an atom of $B$.

Corollary 4.2. Let $C$ be a sublattice of $L$ that generates $B$. If $0 \notin L$ and $R$-generates $B$, then $C$ does not have a smallest element and hence, $C R$-generates B.

The proof of the next proposition is similar to the proof in Gratzer (1971, Lemma 27) and will be omitted.

Proposition 4.3. Let $C$ be a chain in $L$ that generates $B$. Then

(i) $C \cup\{0\}$ is a maximal chain in $B$;

(ii) if $L$ does not have a smallest element and $a \in L$, then $a \geqq c$ for some $c \in C$.

As an immediate consequence of (i) of this proposition, we have

Corollary 4.4. If $C$ is a chain in $L$ and $C$ generates $B$, then $C^{0}$ is a maximal chain in $L$.

Corollary 4.5. If $C$ is a chain in $L, L R$-generates $B$, and $C$ generates $B$, then $C$ is strongly maximal in $L$. 
Proof. Let $\phi$ be a homomorphism of $L$ onto a distributive lattice $K$ and let $D$ be a generalized Boolean lattice $R$-generated by $K$. Then by Gratzer (1971, Corollary 7), $\phi$ can be extended to a homomorphism $\sigma$ of $B$ onto $D$. Since $C$ generates $B, C \phi=C \sigma$ generates $D$. By Corollary $4.4,(C \phi)^{0}$ is a maximal chain in $K$. Thus, $C$ is strongly maximal in $L$.

THEOREM 4.6. Let $B$ be $R$-generated by $L$ and $C$ be a chain in $L$.

(i) If $0 \in L$, then $C$ generates $B$ if and only if $C$ is strongly maximal in $L$.

(ii) If $0 \notin L$, then $C$ generates $B$ if and only if $C$ is strongly maximal in $L$ and $[C]_{L}=L$.

Proof. If $C$ generates $B$, then by Corollary $4.5, C$ is strongly maximal in $L$. If $0 \notin L$, then $L$ does not have a smallest element and so by Proposition 4.3, $[C]_{L}=L$. Thus, we have proven the only if part in both (i) and (ii).

(i) Suppose that $0 \in L$ and that $C$ does not generate $B$. Then if $A$ is the subring of $B$ generated by $C, A \neq B$. If $A$ is an ideal of $B$, then $A \subseteq P$ for some $P \in \mathscr{P}(B)$. Define $\phi$ from $L$ into $C_{2}$ by

$$
a \phi= \begin{cases}0 & \text { if } a \in L \cap P, \\ 1 & \text { if } a \in L \backslash P .\end{cases}
$$

Then $\phi$ is a homomorphism of $L$ onto $C_{2}$ and $C \phi=\{0\}$. Therefore, $C$ is not strongly maximal in $L$. Suppose that $A$ is not an ideal of $B$. Then there exists $x \in B \backslash A$ such that $x<z$ for some $z \in A$ and $z \leqq c$ for some $c \in C$. By Proposition 3.8, there exists $P, Q \in \mathscr{P}(B)$ such that $x \in Q \backslash P, P \cap A=Q \cap A$, and $c \notin P \cup Q$. By Theorem 3.6, $P \cap L \neq Q \cap L$. Now as in Gratzer (1971, p. 115) define $\phi$ from $L$ into $C_{2} \times C_{2}$ by

$$
a \phi= \begin{cases}(0,0) & \text { if } a \in L \cap P \cap Q, \\ (1,0) & \text { if } a \in(L \cap Q) \backslash P, \\ (0,1) & \text { if } a \in(L \cap P) \backslash Q, \\ (1,1) & \text { if } a \in L \backslash(P \cup Q) .\end{cases}
$$

Since $P \cap A=Q \cap A$, it follows that $C \subseteq(P \cap Q) \cup(L \backslash P \cup Q))$ and so $C \phi \subseteq\{(0,0),(1,1)\}$. Now, $c, 0 \in L$, hence, $\{(0,0),(1,1)\} \subseteq L \phi$ (note, this is the first place that we have used the hypothesis that $0 \in L$ ), and since $P \cap L \neq Q \cap$ $L, L \phi$ has at least three elements. Again, we have that $C$ is not strongly maximal in $L$.

(ii) Suppose that $0 \notin L,[C)_{L}=L$, and that $C$ does not generate $B$. Then, as in the proof of (i), $A$ is not an ideal of $B$ and there exists $x \in B \backslash A$ such that 
$x \leqq c$ for some $c \in C$. If $x \in E$, then $x+c \notin E, x+c \in B \backslash A$, and $x+c<c$. By Byrd, Mena and Troy (1975, Corollary 2.2) $x+c \geqq a$ for some $a \in L$. If $d \in C$ such that $d \leqq x$, then $d<x+c$. Thus, by Proposition 3.8, there exists $P, Q \in$ $\mathscr{P}(B)$ such that $x+c \in Q \backslash P, \quad c \in P \cup Q, \quad P \cap A=Q \cap A, \quad$ and $P \cap Q \cap L \neq \square$. Define $\phi$ from $L$ into $C_{2} \times C_{2}$ as in (i). Then again, $C \phi \subseteq$ $\{(0,0),(1,1)\}$ and $(1,1) \in L \phi$. Since $P \cap Q \cap L \neq \square,(0,0) \in L \phi$ and by Theorem 3.6, $P \cap L, Q \cap L$ are distinct elements of $\mathscr{P}(L)$. Thus, again $L \phi$ has at least three elements and so $C$ is not strongly maximal in $L$.

An immediate consequence of the theorem and Proposition 4.3 is

Corollary 4.7. Let $B$ be $R$-generated by $L$ and $C$ be a chain in $L$.

(i) If $0 \in L$ and $C$ is strongly maximal in $L$, then $C \cup\{0\}$ is a maximal chain in $B$.

(ii) If $0 \notin L, C$ is strongly maximal in $L$, and $[C)_{L}=L$, then $C \cup\{0\}$ is a maximal chain in $B$.

\section{Examples}

The first example serves to illustrate several points.

Example 5.1. Let $B=\{x \mid x \in p(N), x$ is finite or $N \backslash x$ is finite $\}$. Then $B$ is a Boolean sublattice of $p(N)$. If $L=\{a \mid a \in p(N)$ and $N \backslash a$ is finite $\}$, then $L$ is a sublattice of $B, L$ does not have a smallest element, and $B$ is $R$-generated by $L$.

If $J=\{a \mid a \in L$ and $1 \notin a\}$, then $J \in \mathscr{P}(L), L \backslash J=\{a \mid a \in L$ and $1 \in a\}$, and $N, N \backslash\{2\} \in L \backslash J$. Thus, $\{2\}=N+N \backslash\{2\} \in E_{L \backslash J}$. Also, $N \backslash\{1\} \in J$ and so $\{2\} \in(J]_{B}$. Hence, $(J]_{B}+E_{L \backslash J}$ is not the direct sum of $(J]_{B}$ and $E_{L \backslash J}$.

Next let $x_{1}=\square$, for $n>1$, let $x_{n}=\{2, \cdots, n\}$, and for $m \in N$, let $c_{m}=$ $N \backslash x_{m}$. Then $C=\left\{c_{n} \mid n \in N\right\}$ is a chain in $L$. Now $N \backslash\{1\} \in L$ and for each $n$, $c_{n} \equiv N \backslash\{1\}$. Thus, by Proposition 4.3 (ii), $C$ does not generate $B$.

We now show that $C$ is strongly maximal in $L$. Note that if $a_{1}, a_{2} \in L$ with $a_{1} \subseteq a_{2}$ and $a_{2} \backslash a_{1} \subseteq x_{n}$ for some $n$, then $a_{1} \wedge c_{n}=a_{2} \wedge c_{n}$. Let $\phi$ be a homomorphism of $L$ onto a distributive lattice $K$ and let $a \in L$ such that $a \phi \notin C \phi$ and $C \phi \cup\{a \phi\}$ is a chain in $K$. We show that $a \phi$ is the zero of $K$. Now $c_{1} \phi>a \phi$ as $c_{1}$ is the largest element of $L$. Let $M=\left\{n \mid c_{n} \phi>a \phi\right\}$. Then $M$ is nonempty and either $M$ is finite or $M=N$. Suppose (by way of contradiction) that $m$ is the largest element of $M$. Then $c_{m} \phi>a \phi>c_{m+1} \phi$. If $b=\left(c_{m+1} \vee a\right) \wedge c_{m}$, then $c_{m+1} \leqq$ $b \leqq c_{m}$ and $b \phi=a \phi$. Hence, $c_{m+1}<b<c_{m}$, but this is impossible as $\{d \mid d \in L$ and $\left.c_{m+1}<d<c_{m}\right\}=\square$. Thus, $M=N$.

If $1 \in a$, then $N \backslash a \subseteq x_{m}$ for some $m$. But then $a \geqq c_{m}$, which implies $a \phi \geqq c_{m} \phi$, a contradiction. Hence, $1 \notin a$. Next let $b \in L$ and $\dot{d}=b \wedge a$. Then 
$1 \notin d$. Now, $a \backslash d$ is finite and so $a \backslash d \subseteq x_{m}$ for some $m$. Then, as noted above, we have $d \wedge c_{m}=a \wedge c_{m}$. Thus,

$$
a \phi=a \phi \wedge c_{m} \phi=\left(a \wedge c_{m}\right) \phi=\left(d \wedge c_{m}\right) \phi=d \phi \wedge c_{m} \phi \leqq d \phi \leqq b \phi .
$$

It now follows that $a \phi$ is the smallest element of $K$ and so $(C \phi)^{0}$ is maximal in $K$. Hence, $C$ is strongly maximal in $L$, but $C$ does not $R$-generate $B$ as asserted in Gratzer (1971, Theorem 28).

Finally, since $C$ is strongly maximal in $L, C$ is a maximal chain in $L$. But $C^{0}$ is not a maximal chain in $B$, for $\{1\} \in B \backslash L$ and $c \cup\{\{1\}\}$ is a chain in $B$. This shows that the conditions given in (ii) of Corollary 4.7 cannot be weakened.

EXAMPLE 5.2. Let $Z$ denote the set of integers, for $n \in Z$ let $(n]$ denote the ideal of $Z$ generated by $n$, let $F$ denote the collection of finite subsets of $Z$, let $L=\{(n] \mid n \in Z\}$, and let $B=F \cup\{(n] \cup x \mid n \in Z$ and $x \in F\}$. Then $B$ is a Boolean sublattice of $p(Z)$. Moreover, $L$ is linearly ordered and $R$-generates $B$, and $F$ is the ideal of $B$ evenly generated by $L$.

If $G=\{x \mid x \in F$ and $0 \notin x\}$, then $G$ is a maximal ideal of $F$ and the index of $G$ in $B$ is 4. Thus, $B / G=\{G, G+\{0\}, G+(-1], G+(0]\}$ and $B / G$ is isomorphic to the four element Boolean lattice $C_{2} \times C_{2}$. If $\phi$ is the natural mapping of $B$ onto $B / G$, then $L \phi$ does not $R$-generate $B \phi$ as is suggested in the proof of Gratzer (1971 Theorem 28). It is easily seen that $L \phi$ is strongly maximal in $B / G$ and, as noted above $L \phi$ does not $R$-generate $B / G=B \phi$, showing that the if portion of Gratzer (1971, Theorem 28 ) is not valid. Also, $B / G$ is the smallest sublattice of itself containing $(L \phi)^{\circ}$ and closed under the formation of relative complements. Thus, the if portion of Gratzer (1971, Lemma 15) is not true.

Finally, $B$ is $R$-generated by itself and $L$ is a chain in $B$ that $R$-generates $B$. Thus, by Theorem 4.6 (i), $L$ is strongly maximal in $B$. However, $[L)_{B} \neq B$. Hence, apparently we cannot combine (i) and (ii) of Theorem 4.6 into a single assertion.

\section{References}

R. D. Byrd, R. A. Mena and L. A. Troy (to appear), 'Generalized Boolean Lattices', J. Austral. Math. Soc.

G. Gratzer (1971), Lattice Theory (W. H. Freeman and Company, San Francisco, 1971).

D. C. Makinson (1969), 'On the number of ultrafilters in a Boolean algebra', $Z$. Math. Logik Grundlagen Math. 15, 121-122.

Department of Mathematics

University of Houston

Houston, Texas 77004

U.S.A. 